SHORT PAPER

\title{
Analysis of DQB 1 allele frequencies in pulmonary tuberculosis: preliminary report
}

\author{
A Dubaniewicz, G Moszkowska, Z Szczerkowska, A Hoppe
}

See end of article for

authors' affiliations

Thorax 2003;58:890-891

Correspondence to:

Dr A Dubaniewicz, Department of

Pathophysiology, Medical University of Gdańsk, 80952 Gdańsk, Dębinki 7 str, Poland;

aduban@amedec.amg. gda.pl

Revised version received 8 January 2003

Accepted for publication

10 June 2003
Background: The human leucocyte antigen (HLA) system plays an important role in the modulation of the immune response. An association between HLA and pulmonary tuberculosis (TB) has been examined in several populations but the results have been inconsistent. The aim of this study was to evaluate the correlation of DQB1 alleles with TB patients and healthy controls in the same ethnic group in Poland.

Method: The DQB1 alleles of 38 patients with TB and 58 healthy university staff volunteers were determined by a PCR-SSP low resolution method.

Results: The $D Q B 1 * 05$ allele occurred more frequently ( $p$ adjusted for multiple comparison $=0.002$, $\mathrm{OR}=2.84,95 \% \mathrm{Cl} 1.57$ to 5.15$)$ and the $\mathrm{DQB} 1 * 02$ allele occurred less frequently $(p=0.01, \mathrm{OR}=0.39$, $95 \% \mathrm{Cl} 0.21$ to 0.71 ) in patients with $\mathrm{TB}$ than in controls. The occurrence of $\mathrm{DQB} 1{ }^{*} 03,{ }^{*} 04,{ }^{*} 06$ alleles was similar in the two populations.

Conclusions: The occurrence of specific DQB1 alleles may be linked to susceptibility/resistance to tuberculosis.
$\mathrm{T}$ uberculosis (TB) is still an important world health problem, and it is estimated that about one third of the earth's population has been infected with Mycobacterium tuberculosis. Each year there are $\sim 2$ million deaths from TB. It is still not clear why only approximately one in 10 of those infected progress to active disease during their lifetime when only a minority have a risk factor. ${ }^{1}$

Tuberculosis develops by a complex of environmental factors and genetic susceptibility. The observations of the familial occurrence of TB and the description of the disease in monozygotic twins suggest that genetic elements might contribute to determining the course of the infection. It has recently been reported that mutations in genes encoding natural resistance associated macrophage protein l (NRAMP$1)$, interferon- $\gamma$ receptor, or one component of the interleukin- 12 receptor might affect the susceptibility to TB. ${ }^{1}$

The main stages of cell reactivity-presentation of antigen, phagocytosis, cooperation with $\mathrm{T}$ and $\mathrm{B}$ lymphocytes, or bactericidal activity-depend on the specificity of the HLA system. ${ }^{1}$ Since the DR alleles play an important role in the modulation of the immune response, a possible association between DQB alleles and TB has been examined in Asian and American ethnic groups, but the results have been inconsistent..$^{2-7}$ Despite the rising incidence of TB in Europe, no such analysis has yet been carried out in European white populations. A study was therefore undertaken to evaluate the occurrence of DQBI alleles in TB patients and healthy controls in the same ethnic group in Poland.

\section{METHODS}

\section{Patients}

With the approval of the Independent Bioethics Committee for Scientific Researches, Medical University of Gdańsk, Poland, 38 unrelated patients ( 23 men) of mean age 40 years (range 22-68) with newly detected active pulmonary TB were studied at the Pulmonological Hospital, Sopot between July and December 2002. The diagnosis of TB was confirmed in all patients by the presence of acid-fast bacilli in sputum smears and by positive sputum culture of $M$ tuberculosis strains. Patients were classified according to clinical stage and appearance on the initial chest radiograph (infiltrates with cavitation in one or two lung zones). A positive PPD skin test was an additional diagnostic criterion. Patients who did not respond to first line treatment (rifampin, isoniazid, ethambutol, pyrazinamide) were excluded from the study.

\section{Controls}

Fifty eight unrelated individuals (32 men) of mean age 42 years (range 27-60) without clinical, physical, or laboratory evidence of TB formed the control group. A negative PPD skin test was used to confirm that they did not have TB.

Patients and controls were excluded from the study if they had a family history of TB or other related diseases. Those of different socioeconomic status and ethnic background were also excluded from the study. The Polish population is a relatively homogenous white ethnic group. All patients and controls had been vaccinated with BCG (bacillus CalmetteGuerin).

\section{HLA typing}

Genomic DNA was extracted from $10 \mathrm{ml}$ peripheral blood from each individual using the salt extraction method. DQB 1 typing was performed using sequence specific amplification polymerase chain reaction with sequence specific primer (PCR-SSP) according to the method described by Olerup and coworkers. ${ }^{8}$ The DQBl primers were supplied by DYNAL in the DYNAL DQ “low resolution" SSP standard kit.

\section{Analysis of data}

Data were analysed with STATISTICA for Windows Version 6.0 (StatSoft Inc,USA). Group comparisons were made using the $\chi^{2}$ test after the Bonferroni correction $(p \leqslant 0.05)$. The odds ratio (OR) was calculated with $95 \%$ confidence intervals (CI).

\section{RESULTS}

A comparison of the frequencies of the DQBl alleles in the patients with TB and in the control population is shown in table 1.

The results indicate that the $\mathrm{DQB1}{ }^{*} 05$ allele was detected more frequently in patients with TB than in control subjects 
Table 1 Frequency of DQB1 alleles in patients with pulmonary tuberculosis (TB) and controls

\begin{tabular}{|c|c|c|c|}
\hline \multirow[b]{2}{*}{ DQB 1 alleles } & \multicolumn{2}{|c|}{$\begin{array}{l}\text { No (\%) allele positive } \\
\text { individuals }\end{array}$} & \multirow[b]{2}{*}{$\begin{array}{l}\text { Odds ratio } \\
(95 \% \mathrm{CI})\end{array}$} \\
\hline & $\begin{array}{l}\text { Controls } \\
(n=58)\end{array}$ & $\begin{array}{l}\text { TB patients } \\
(\mathrm{n}=38)\end{array}$ & \\
\hline $\mathrm{DQB} 1{ }^{*} 02+$ & $27(47 \%)$ & $10(26 \%)$ & $0.39(0.21$ to 0.71$)$ \\
\hline $\mathrm{DQB1}{ }^{*} 03$ & $39(67 \%)$ & $28(73 \%)$ & $1.33(0.72$ to 2.44$)$ \\
\hline $\mathrm{DQB} 1{ }^{*} 04$ & 0 & $2(3 \%)$ & 0 \\
\hline $\mathrm{DQB} 1{ }^{*} 05 \ddagger$ & $15(26 \%)$ & $19(50 \%)$ & $2.84(1.57$ to 5.15$)$ \\
\hline $\mathrm{DQB} 1{ }^{* 06}$ & $28(48 \%)$ & $18(47 \%)$ & $0.96(0.55$ to 1.67$)$ \\
\hline
\end{tabular}

$\dagger p=0.002, p c$ ( $p$ adjusted for multiple comparison $)=0.01 ; \neq p=0.0005$, $\mathrm{pc}=0.002$.

$\left(50 \% \quad v \quad 26 \% ; \quad \chi^{2}=12.12 ; \quad \mathrm{p}=0.0005 ; \quad \mathrm{pc}=0.002\right)$. The DQB $1 * 02$ allele occurred less frequently in patients with TB than in healthy controls $\left(26 \% \vee 47 \% ; \chi^{2}=9.51 ; p=0.002\right.$; $\mathrm{pc}=0.01)$. There was no difference between the two groups in the frequency of the remaining alleles $\left(\mathrm{DQB1}{ }^{*} 03\right.$, $\mathrm{DQB1}^{*} 04$, and $\mathrm{DQBI}^{*} 06$ ).

\section{DISCUSSION}

There have been few reports of the correlation between the frequency of DQBI alleles and the susceptibility to pulmonary $\mathrm{TB}^{2-7} \mathrm{~A}$ significant association was found between the occurrence of DQB1*0501, ${ }^{*} 0502$ and ${ }^{*} 0601$ alleles and TB in subjects from North India. ${ }^{2}$ In South India the results of studies on the association of DQB1 alleles with the development of clinical TB have been inconsistent ${ }^{3}{ }^{4}$; Ravikumar et $a l^{3}$ found a higher frequency of $\mathrm{DQB}{ }^{*} 0601$ in those with TB while Sanjeevi et $a l^{4}$ found no correlation between $\mathrm{TB}$ and DQB1 alleles. In Mexican patients with TB a significant positive association with DQB1*0501 was reported by TeranEscandon et al, ${ }^{5}$ whereas in a Thai population DQB1* 0502 was found more frequently in subjects with TB. ${ }^{6}$ In Cambodia an association was found between $\mathrm{TB}$ and $\mathrm{DQB}{ }^{*} 0503$ but not with $\mathrm{DQB1}{ }^{*} 0501$ or $\mathrm{DQB1}{ }^{*} 0601 .^{7}$ A negative association between the presence of $M$ tuberculosis and DQB1*0301 in Thai subjects and $\mathrm{DQB1}{ }^{*} 0402$ in Mexicans was noted. ${ }^{5} 6$

The finding in our study of a high frequency of DQB $1 * 05$ in patients with TB is in agreement with that of previous studies in many populations. ${ }^{25-7}$ However, we were not able to confirm a positive association with $\mathrm{DQB}{ }^{*} 06$ or a negative association with $\mathrm{DQB1}{ }^{*} 03$ or $\mathrm{DQB1}{ }^{*} 04$ alleles as has been reported in other populations. ${ }^{356}$ There have been no reports on the frequency of the $\mathrm{DQB1}{ }^{*} 02$ allele in $\mathrm{TB}$.

The discrepancies in the results may be caused by different methods and/or the high degree of polymorphism of the DQB1 allele in different ethnic groups, in which significant geographical variations have been observed. Stern et al ${ }^{9}$ showed that the DQB1*0503 allele encodes a change in the amino acid position 57 of the $\beta$ chain which influences the charge in the putative peptide binding pocket (P9) of the DQ molecule. The negatively charged P9 binding pocket may bind $\mathrm{TB}$ antigens less effectively or elicit a diminished immunogenic response. ${ }^{9}$ The findings of Goldfeld et $a l^{7}$ support the evidence for an association between a specific $\mathrm{DQB}{ }^{*} 0503$ allele and progressive clinical TB. Geluk et $a l^{10}$ recently identified three new $\mathrm{DQAl}^{*} 0301 / \mathrm{DQB1}{ }^{*} 0302$ restricted $\mathrm{T}$ cell epitopes of mycobacterial heat shock protein 65 which mounted an efficient response to $M$ tuberculosis. Epidemiological and experimental studies suggest that the high degree of molecular diversity in HLA molecules influences the variability in the human response to $M$ tuberculosis.

\section{ACKNOWLEDGEMENTS}

This study was funded by the Polish State Committee for Scientific Researches grant no. 3PO5B 15522.

\section{Authors' affiliations}

A Dubaniewicz, A Hoppe, Department of Pathophysiology, Medical University of Gdańsk, Poland

G Moszkowska, Department of Immunopathology, Medical University of Gdańsk

Z Szczerkowska, Division of Forensic Medicine, Medical University of Gdańsk

\section{REFERENCES}

1 Schluger NW, William NR. The host immune response to tuberculosis. Am J Respir Crit Care Med 1998;157:679-91.

2 Rani R, Mukherjee R, Stastny P. Diversity of HLA-DR2 in North Indians:the changed scenario after the discovery of $D R B 1 * 1506$. Tissue Antigens 1998;52:147-52.

3 Ravikumar M, Dheenadhayalan V, Rajaram K, et al. Associated of HLADRB1, DQB1 and DPB1 alleles with pulmonary tuberculosis in south India. Tuber Lung Dis 1999;79:309-17.

4 Sanjeevi CB, Narayanan PR, Prabakar R, et al. No association or linkage with HLA-DR2 or DQ genes in South Indians with pulmonary tuberculosis. Tubercle 1992;73:280-4.

5 Teran-Escandon D, Teran-Ortiz L, Camarena-Olvera A, et al. Human leukocyte antigen-associated susceptibility to pulmonary tuberculosis: molecular analysis of class II alleles by DNA amplification and oligonucleotide hybridization in Mexican patients. Chest 1999;115:428-33.

6 Vebaesya S, Chierakul N, Luangtrakool K, et al. Associations of HLA class II alleles with pulmonary tuberculosis in Thais. Eur J Immunogenet 2002;29:431-4.

7 Goldfeld AE, Delgado JC, Bozon VB, et al. Association of an HLA-DQ allele with clinical tuberculosis. JAMA 1998;279:226-8.

8 Olerup O, Aldener A, Fogdell A. HLA-DQB land HLA-DQA1 typing by PCR amplifi-cation with sequence-specific primers (PCR-SSP) in 2 hours. Tissue Antigens 1993;41:119-34.

9 Stern $\mathrm{U}$, Brown JH, Jardetsky TS, et al. Crystal structure of the human class II MHC protein HLA-DR1 complexed with an influenza virus peptide. Nature 1994;368:215-21.

10 Geluk A, Taneja $V$, vanMeijgaarden KE, et al. Identification of HLA class IIrestricted determinants of mycobacterium tuberculosis-derived proteins by using HLA-transgenic, class II-deficient mice. Immunology 1998;95: 10797-802. 